\title{
Absolute Stability of Multi-DOF Multi-lateral Haptic Systems
}

\author{
Jian Li, Student Member, IEEE, Mahdi Tavakoli, Member, IEEE, and Qi Huang, Senior Member, IEEE
}

\begin{abstract}
Multi-degree-of-freedom (DOF) multi-lateral haptic systems involve teleoperation of several robots in physical environments by several human operators or collaborative interaction of several human operators in a virtual environment. An $m$-DOF $n$-lateral haptic system can be modeled as an $n$-port network where each port (terminal) connects to a termination defined by $m$ inputs and $m$ outputs. The stability analysis of such systems is not trivial due to dynamic coupling across the different DOFs of the robots, the human operators, and the physical/virtual environments, and unknown dynamics of the human operators and the environments exacerbate the problem.

Llewellyn's criterion only allows for absolute stability analysis of 1-DOF bilateral haptic systems $(m=1, n=2)$, which can be modeled as two-port networks. The absolute stability of a general $m$-DOF bilateral haptic system where $m>1$ cannot be obtained from $m$ applications of Llewellyn's criterion to each DOF of the bilateral system. Also, if we were to use Llewellyn's criterion for absolute stability analysis of a general 1-DOF $n$ lateral haptic system where $n>2$, we would need to couple $n-2$ terminations of the $n$-port network to (an infinite number of) known impedances in order to reduce it to an equivalent two-port network; this is a cumbersome process that involves an infinite number of applications of Llewellyn's criterion. In this paper, we present a straightforward and convenient criterion for absolute stability analysis of a class of $m$-DOF $n$-lateral haptic systems for any $m \geq 1$ and $n \geq 2$. As case studies, a 1-DOF trilateral and a 2-DOF bilateral haptic system are studied for absolute stability with simulations and experiments confirming the theoretical stability conditions.
\end{abstract}

Index Terms-Multi-port network, multi-lateral haptic system, absolute stability.

\section{INTRODUCTION}

Multi-lateral haptic systems have recently found applications in tele-medicine [1], [2], cooperative robotics for humanrobot lunar exploration [3], and multi-robot systems [4], [5]. An $m$-DOF $n$-lateral haptic system can be modeled as an $n$-port network where each port (terminal) connects to an $m$-DOF termination. In the special case of $m=1$ and $n=2$, this is a bilateral teleoperation system modeled as a two-port network. Multi-port networks are widely used in other applications such as radio-frequency and microwave circuits to analyze their absolute stability (sometimes called unconditional stability) [6], [7].

For a teleoperation system consisting of a teleoperator comprised of master(s), slave(s) and controllers coupled to terminations consisting of human operator(s) and environment(s),

This research was supported by the Natural Sciences and Engineering Research Council (NSERC) of Canada, by the Natural Science Foundation of China (NFSC, Grant No. 51277022), and by the China Scholarship Council (CSC) under grant [2011]3005.

Jian $\mathrm{Li}$ is with the School of Energy Science and Engineering, University of Electronic Science and Technology of China, Chengdu, Sichuan, 611731 China. Jian Li and Mahdi Tavakoli are with the Department of Electrical and Computer Engineering, University of Alberta, Edmonton, Alberta, T6G 2V4 Canada. Qi Huang is with the School of Energy Science and Engineering, University of Electronic Science and Technology of China, Chengdu, Sichuan, 611731 China.

E-mail: uajian@gmail.com, mahdi.tavakoli@ualberta.ca, closed-loop stability is critical for safe and effective teleoperation. Investigation of teleoperation system stability using common closed-loop stability analysis tools in the control systems literature is not possible because the models of the human operator(s) and the environment(s) are usually unknown, uncertain, and/or time-varying. However, research has shown that it is still possible to draw stability conditions for a haptic teleoperation system under unknown "terminations" as long as they are passive. These stability conditions can be categorized as passivity and absolute stability criteria.

For stability analysis of 1-DOF $n$-lateral haptic systems, passivity is used in [8], [9] for $n=2$, in [10], [11] for $n=3$, and in [12] for any $n \geq 2$. Specifically, in [8], Raisbeck's method is useful as a passivity criterion for 1DOF bilateral teleoperation systems based on the immittance matrix of the teleoperator. Shahbazi et al. in [10] performed stability analysis for a dual-user (trilateral) teleoperation system based on the passivity definition for a three-port network. In [11], Panzirsch et al. proposed a time-domain passivity observer/passivity controller approach for a dual-user (trilateral) teleoperation system. In [12], Mendez and Tavakoli presented a criterion (necessary and sufficient) for passivity of general $n$-port networks, which can model 1-DOF $n$-lateral haptic systems.

Passivity of a multi-port network is a conservative condition for its coupled stability. A less conservative condition, absolute stability is discussed in [13], [14] for $n=2$, in [15], [16] for $n=3$, and in [17] for any $n \geq 2$. Specifically, Llewellyn in [14] proposed an absolute stability criterion for two-port networks, which model 1-DOF bilateral teleoperation systems, based on the immittance matrix of network (the teleoperator). In [15], Khademian et al. analyzed absolute stability of a dualuser (trilateral) teleoperation system by reducing the three-port network to an equivalent two-port network, paving the way for the applications of Llewellyn's criterion. Li et al. in [16] presented an absolute stability criterion for a class of trilateral haptic systems. In [17], Ku studied $n$-port network stability if the impedance matrix of the $n$-port network conforms to the tri-diagonal Jacobian form. The above research only addresses the absolute stability analysis of 1-DOF haptic teleoperators.

In past research, for stability analysis of multi-DOF bilateral [18] and tri-lateral haptic systems [19], the multi-DOF systems are decoupled to 1-DOF systems. Then, various stability criteria for 1-DOF $n$-lateral haptic systems are used. This poses difficulties in terms of decoupling a coupled haptic system especially because the human operator(s) and the environment(s) terminations are also coupled themselves. In this paper, we present a criterion to analyze the absolute stability of multi-DOF multi-lateral haptic systems directly and without a need for decoupling. As a case study, we consider a 2-DOF bilateral haptic system and use the proposed absolute stability criterion to design stabilizing controllers for the system.

The rest of the paper is organized as follows: The next 
section gives mathematical definitions and lemmas for analysis of absolute stability. Section III introduces simple motivating examples to show that the absolute stability criteria for 1-DOF bilateral haptic systems fail to analyze the absolute stability of $m$-DOF bilateral haptic systems. Next, in Section IV, the proposed absolute stability criterion for $m$-DOF $n$-lateral networks is derived. Then, as a case study to show how the resulting absolute stability criterion can be utilized, in Section V, a 1-DOF trilateral haptic system is considered, the absolute stability conditions in terms of system parameters including controller gains are found, and simulations to verify the validity of the calculated absolute stability conditions are conducted. In another case study, in Section VI, a 2-DOF bilateral teleoperation system with position-position control is considered, the absolute stability conditions in terms of system parameters including controller gains are found, and experiments to verify the validity of the calculated absolute stability conditions are presented. Section VII contains concluding remarks.

\section{Mathematical Preliminaries}

Notation 1. $a$ is a scalar, $A$ is a vector, $\mathbf{A}$ is a matrix, and $\mathcal{A}$ is a block matrix (i.e., with matrix elements).

Definition 1. [20] A multi-port network is passive if the total energy delivered to the network at its ports is non-negative.

Property 1. [21] A gyration operator, which transforms one immittance matrix to another, preserves the passivity property.

Definition 2. [22] A $n \times n$ proper rational transfer matrix $\mathbf{G}(\mathbf{s})$ is positive real if

i) Poles of all elements of $\mathbf{G}(\mathbf{s})$ are in $\operatorname{Re}[s] \leq 0$,

ii) Any pure imaginary pole $j \omega$ of any element of $\mathbf{G}(\mathbf{s})$ is a simple pole and the residue matrix $\lim _{s \rightarrow j \omega}(s-j \omega) \mathbf{G}(\mathbf{s})$ is positive semidefinite Hermitian,

iii) For all real $\omega$ for which $j \omega$ is not a pole of any element of $\mathbf{G}(\mathbf{s})$, the matrix $\mathbf{G}(j \omega)+\mathbf{G}^{T}(-j \omega)$ is positive semidefinite.

Property 2. [23] The Hermitian part of a symmetric matrix is a real matrix. A real matrix is positive semidefinite if its principal minors are all nonnegative.

Lemma 1. [22] A linear time-invariant minimal realization model with transfer matrix $\mathbf{G}(\mathbf{s})$ is passive if $\mathbf{G}(\mathbf{s})$ is positive real.

Definition 3. [24] A multi-port network is absolutely stable if the coupled system remains bounded-input bounded-output stable under all possible passive terminations. Otherwise, it is potentially unstable.

Lemma 2. [25] Let $\mathbf{Z}=\mathbf{Z}^{\mathrm{T}}$ be the impedance matrix of a reciprocal n-port network. Then, the network is passive if and only if it is absolutely stable.

Lemma 3. [26] Let $\mathbf{Z}_{1}$ and $\mathbf{Z}_{2}$ be the impedance matrices of two n-port networks. Then, if $\mathbf{Z}_{1}$ and $\mathbf{Z}_{2}$ possess identical principal minors of all orders, then $\mathbf{Z}_{1}$ is absolutely stable if and only if $\mathbf{Z}_{2}$ is absolutely stable.

\section{MOTIVATION}

Llewellyn's criterion has been used to analyze the absolute stability of 1-DOF bilateral teleoperation systems. In the following, using two examples, we show why it cannot be used for coupled $m$-DOF $(m>1)$ bilateral teleoperation systems. For absolute stability, both terminations of the twoport network need to be passive. For a coupled 2-DOF bilateral teleoperation system, consider the following termination for its first port:

$$
\mathbf{T}_{1}=\left[\begin{array}{cc}
\frac{8}{s+3} & -\frac{5}{s+1} \\
-\frac{5}{s+1} & \frac{1}{s+3}
\end{array}\right]
$$

According to Definition 2 and Property 2, we find that although the terminations $\frac{8}{s+3}$ and $\frac{1}{s+3}$ along each of the first two DOFs are passive, the coupled 2-DOF termination $\mathbf{T}_{1}$ is non-passive. Therefore, viewing the termination impedances along each of the DOFs separately can result in misleading results in terms of absolute stability.

As another case, consider a coupled 2-DOF bilateral teleoperator modeled as

$$
\left[\begin{array}{l}
F_{h} \\
F_{e}
\end{array}\right]=\mathcal{Z}\left[\begin{array}{l}
V_{h} \\
V_{e}
\end{array}\right]
$$

where $F_{h}=\left[f_{h x}, f_{h y}\right]^{T}, F_{e}=\left[f_{e x}, f_{e y}\right]^{T}, V_{h}=\left[v_{h x}, v_{h y}\right]^{T}$, $V_{e}=\left[v_{e x}, v_{e y}\right]^{T}$, and

$$
\begin{aligned}
& \mathcal{Z}=\left[\begin{array}{ll}
\mathbf{Z}_{11} & \mathbf{Z}_{12} \\
\mathbf{Z}_{21} & \mathbf{Z}_{22}
\end{array}\right] \\
& =\left[\begin{array}{cc:c|c}
\frac{9}{s+3} & -\frac{5}{s+5} & -\frac{1}{s+1} & -\frac{1}{s+2} \\
-\frac{5}{s+5} & \frac{9}{s+3} & -\frac{1}{s+3} & -\frac{1}{s+1} \\
\hdashline-\frac{1}{s+1} & -\frac{1}{s+3} & \frac{1}{s+3} & -\frac{9}{s+1} \\
\hdashline-\frac{1}{s+2} & -\frac{1}{s+1} & -\frac{9}{s+1} & \frac{1}{s+3} \\
\hdashline----1 & & ---
\end{array}\right]
\end{aligned}
$$

Assume the terminations of this teleoperator are always passive. For using Llewellyn's criterion once along the $x$ direction and once along the $y$ direction, we have to consider the following two subsystems of (2):

$$
\left[\begin{array}{c}
f_{h x} \\
f_{e x}
\end{array}\right]=\mathbf{Z}_{x}\left[\begin{array}{l}
v_{h x} \\
v_{e x}
\end{array}\right], \quad\left[\begin{array}{l}
f_{h y} \\
f_{e y}
\end{array}\right]=\mathbf{Z}_{y}\left[\begin{array}{l}
v_{h y} \\
v_{e y}
\end{array}\right]
$$

where

$$
\mathbf{Z}_{x}=\mathbf{Z}_{y}=\left[\begin{array}{cc}
\frac{9}{s+3} & -\frac{1}{s+1} \\
-\frac{1}{s+1} & \frac{1}{s+3}
\end{array}\right]
$$

While the subsystems involving $\mathbf{Z}_{x}$ and $\mathbf{Z}_{y}$ always satisfy Llewellyn's criterion (see Appendix for Llewellyn's criterion), as shown next, the coupled 2-DOF teleoperator (2) is not absolutely stable. In general, for checking the absolute stability of a two-port network such as a bilateral teleoperator, the port \#2 (environment port) can be connected to passive terminations while the input energy at the port \#1 (operator port) is measured. The bilateral teleoperator is absolutely stable if and only if, at all times $t>0$, we have [27]:

$$
E_{s}(t)=\int_{0}^{t} F_{h}^{T}(\tau) V_{h}(\tau) \mathrm{d} \tau \geq 0 .
$$

Similarly, the subsystems involving $\mathbf{Z}_{x}$ is absolutely stable if and only if, at all times $t>0$, we have

$$
E_{s}(t)=\int_{0}^{t} f_{h x}(\tau) v_{h x}(\tau) \mathrm{d} \tau \geq 0 .
$$

As shown in Figure 1, which plots $E_{s}(t)$ for the teleoperator (2) (solid) and each of the two subsystems (4) (dash-dot), 


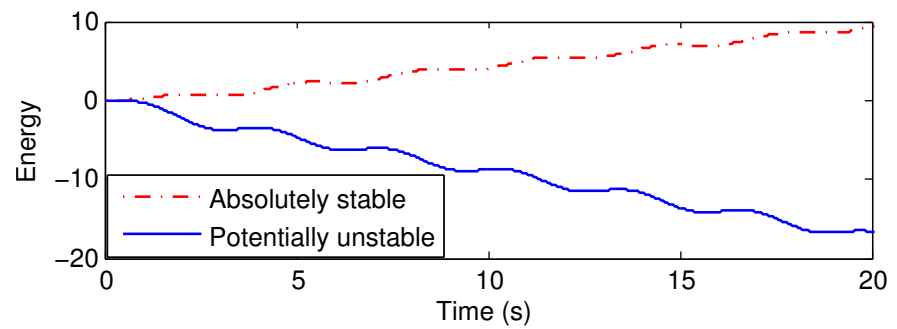

Figure 1. Simulation results for analysis of absolute stability of the 2DOF bilateral teleoperator (3). While the subsystems in (4) always satisfy Llewellyn's criterion as evidenced by the nonnegative energy plot (dashdot), the coupled 2-DOF teleoperator in (3) is actually potentially unstable as evidenced by the negative energy plot (solid).

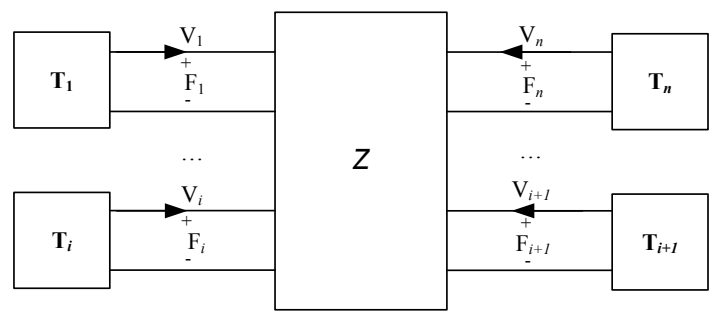

Figure 2. An $n$-port network where each port (terminal) connects to an $m$ DOF termination.

simulations confirmed that each of the two 1-DOF subsystems are absolutely stable while the 2-DOF teleoperator is not absolutely stable (i.e., is potentially unstable). From the above two examples, it is clear that for a 2-DOF haptic system, using Llewellyn's criterion twice in each DOF is not useful as it ignores the coupling that may exist in the terminations (i.e., human operators and environments) and the teleoperator. While we showed that 1-DOF $n$-lateral teleoperator absolute stability analysis methods do not work for $m$-DOF $n$-lateral teleoperators for the special case of $n=2$, the same holds for any $n>2$. To the best of our knowledge, no work has been done on direct absolute stability analysis of $m$-DOF $(m \geq 2)$ $n$-lateral coupled teleoperators. Motivated by these facts, we propose a new absolute stability criterion of $m$-DOF $n$-lateral haptic systems.

\section{Main Result: An Absolute Stability CRiterion FOR MUlti-DOF Multi-LATERAl Haptic Systems}

An $m$-DOF $n$-lateral teleoperation system can be modeled as an $n$-port network where each port (terminal) connects to an $m$-DOF termination as shown in Figure 2. The network impedance model will be

$$
\mathcal{F}=\mathcal{Z V}
$$

where

$$
\begin{aligned}
& \mathcal{F}=\left[F_{1}, F_{2}, \cdots, F_{n}\right]^{T} \\
& \mathcal{V}=\left[V_{1}, V_{2}, \cdots, V_{n}\right]^{T}
\end{aligned}
$$

and $F_{i}$ and $V_{i}, i=1,2, \cdots, n$, represent the $m \times 1$ vectors of force and velocity at the $i^{\text {th }}$ port of the network, respectively. The impedance matrix of the network will be

$$
\mathcal{Z}=\left[\begin{array}{cccc}
\mathbf{Z}_{11} & \mathbf{Z}_{12} & \cdots & \mathbf{Z}_{1 n} \\
\mathbf{Z}_{21} & \mathbf{Z}_{22} & \cdots & \mathbf{Z}_{2 n} \\
\vdots & \vdots & \cdots & \vdots \\
\mathbf{Z}_{n 1} & \mathbf{Z}_{n 2} & \cdots & \mathbf{Z}_{n n}
\end{array}\right]_{n \times n}
$$

where $\mathbf{Z}_{i j}, i, j=1,2, \cdots, n$, are $m \times m$ matrices given in (12). On the other hand, the $n$ pairs of $m$-dimensional terminations are represented by

$$
\mathcal{T}=\operatorname{diag}\left[\mathbf{T}_{1}, \mathbf{T}_{2}, \cdots, \mathbf{T}_{n}\right]
$$

where $\mathbf{T}_{i}, i=1,2, \cdots, n$, represents the $m \times m$ impedance matrix of the $i^{\text {th }} m$-dimensional termination.

Theorem 1. An m-DOF n-lateral haptic system with impedance matrix $\mathcal{Z}$ in (11) satisfying the symmetrization conditions

A) $z_{i, j} z_{j, k} z_{k, i}=z_{j, i} z_{k, j} z_{i, k}$, where $i, j, k=1,2, \cdots, m \times n$, $i \neq j \neq k$, and $i \neq k$.

B) $\mathbf{Z}_{\ell \ell}$ is symmetric, where $\ell=1,2, \cdots, n$.

is absolutely stable if and only if

C) The elements of $\mathcal{Z}$ matrix in (12) have no poles in the right-half plane (RHP).

D) Any poles of the elements of the $\mathcal{Z}^{\prime}$ matrix in (13) on the imaginary axis are simple, and the principal minors of the residues matrix of the $\mathcal{Z}^{\prime}$ matrix at these poles are greater than or equal to zero.

$E$ ) For all real values of frequencies $\omega$, the principal minors of the real part of the $\mathcal{Z}^{\prime}$ matrix in (13) are greater than or equal to zero, or equivalently

$$
\begin{aligned}
& \operatorname{Re}\left(z_{i, i}\right) \geq 0, \quad i=1,2, \cdots n \times m \\
& \operatorname{Re}\left(z_{1,1}\right) \operatorname{Re}\left(z_{2,2}\right)-\frac{\left|z_{1,2} z_{2,1}\right|+\operatorname{Re}\left(z_{1,2} z_{2,1}\right)}{2} \geq 0 \\
& \vdots \\
& \operatorname{det}\left(\operatorname{Re}\left(\mathcal{Z}^{\prime}\right)\right) \geq 0
\end{aligned}
$$

Proof. Consider a linear time-invariant system with impulse response $h(t)$. The system's transfer function is the Laplace transform of $h(t)$ defined as

$$
H(s)=\int_{0}^{\infty} h(t) e^{-s t} d t
$$

where $s=\sigma+j \omega . H(s)$ is stable if every bounded input produces a bounded output and this happens if the poles of $H(s)$ have negative real parts. This stability definition is equivalent to the absolute convergence (defined below) of $H(s)$ in the region $\operatorname{Re}(s) \geq 0$. If $h$ is locally integrable, then $H(s)$ is said to converge if the limit $H(s)=\lim _{r \rightarrow \infty} \int_{0}^{r} h(t) e^{-s t} d t$ exists. Also, $H(s)$ is said to converge absolutely if the integral $\int_{0}^{\infty}\left|h(t) e^{-s t}\right| d t$ exists. The set of values of $s$ for which $H(s)$ converges is known as the region of convergence (ROC) and is of the form $\operatorname{Re}(s) \geq a$, where $a$ is a real constant. Importantly, if $H(s)$ converges at $s=s_{0}$, then it automatically converges for all $s$ with $\operatorname{Re}(s)>\operatorname{Re}\left(s_{0}\right)$. The above means that for stability analysis it suffices to focus on the convergence of $H(s)$ when $\operatorname{Re}(s)=0$, i.e., on the $j \omega$ axis. This is sometimes referred to as real-frequency stability. Thus, as a linear timeinvariant system, the stability of an $m$-DOF $n$-lateral haptic system coupled to an $m$-DOF termination at each of its ports needs to only be analyzed for $s=j \omega$.

An $n$-port network is stable if the port currents $I_{1}, I_{2}, \cdots, I_{n}$ are zero under all passive terminations $z_{1}, z_{2}, \cdots, z_{n}$ for ports [25]. In other words, an $n$-port network with an impedance matrix $Z_{n \times n}$ is stable if and only if the equation $\left(Z+Z_{0}\right) \mathbf{I}=0$, where $I=\left[I_{1}, I_{2}, \cdots, I_{n}\right]^{T}$ and $Z_{0}=\operatorname{diag}\left[z_{1}, z_{2}, \cdots, z_{n}\right]$ has only the trivial solution $\mathbf{I}=0$ 


$$
\mathbf{Z}_{i j}=\left[\begin{array}{cccc}
z_{(i-1) m+1,(j-1) m+1} & z_{(i-1) m+1,(j-1) m+2} & \cdots & z_{(i-1) m+1, j m} \\
z_{(i-1) m+2,(j-1) m+1} & z_{(i-1) m+2,(j-1) m+2} & \cdots & z_{(i-1) m+2, j m} \\
\vdots & \vdots & \cdots & \vdots \\
z_{i m,(j-1) m+1} & z_{i m,(j-1) m+2} & \cdots & z_{i m, j m}
\end{array}\right]_{m \times m}
$$

$$
\begin{gathered}
\mathcal{Z}^{\prime}=\left[\begin{array}{cccc}
z_{1,1} & \gamma_{1} \sqrt{z_{1,2} z_{2,1}} & \cdots & \gamma_{m \times n-1} \sqrt{z_{1, m \times n} z_{m \times n, 1}} \\
\gamma_{1} \sqrt{z_{1,2} z_{2,1}} & z_{2,2} & \cdots & \gamma_{2 m \times n-3} \sqrt{z_{2, m \times n} z_{m \times n, 2}} \\
\vdots & \vdots & \cdots & \vdots \\
\gamma_{m \times n-1} \sqrt{z_{1, m \times n} z_{m \times n, 1}} & \gamma_{2 m \times n-3 \sqrt{z_{2, m \times n} z_{m \times n, 2}}} & \cdots & z_{m \times n, m \times n} \\
\text { where, } \quad \gamma_{i}= \pm 1, \quad i=1,2, \cdots, \frac{(m \times n)(m \times n-1)}{2}
\end{array}\right.
\end{gathered}
$$

for every passive choice of $Z_{0}$; this happens if and only if $\operatorname{det}\left(Z+Z_{0}\right) \neq 0$. On the other hand, according to [26], if two $n \times n$ matrices $Z_{1}$ and $Z_{2}$ have identical principal minors of all orders, then

$$
\operatorname{det}\left(Z_{1}+Z_{0}\right)=\operatorname{det}\left(Z_{2}+Z_{0}\right)
$$

for any $Z_{0}=\operatorname{diag}\left[z_{1}, z_{2}, \cdots, z_{n}\right]$. This implies that the stability of two $n$-port networks with impedance matrices $Z_{1}$ and $Z_{2}$ will happen at the same time (Lemma 3).

Now, if there exists a reciprocal $n$-port network with impedance matrix $\mathcal{Z}^{\prime}$ that has the same stability characteristics as the original nonreciprocal $n$-port network with impedance matrix $\mathcal{Z}$, then

$$
\operatorname{det}\left(\mathcal{Z}^{\prime}+\mathcal{T}\right)=\operatorname{det}(\mathcal{Z}+\mathcal{T})
$$

for any passive $\mathcal{T}$ in (14). The above is to hold for any passive $\mathcal{T}$. By induction, it is easy to show that calculating the two determinants and equating the coefficients of $\mathbf{T}_{1}, \mathbf{T}_{2}, \cdots, \mathbf{T}_{n}$ gives the matrix $\mathcal{Z}^{\prime}$ in (13) as well as the symmetrization conditions $\mathrm{A}$ and $\mathrm{B}$.

On the other hand, according to Lemma 2, the reciprocal $n$-port network with impedance matrix $\mathcal{Z}^{\prime}$ is absolutely stable if and only if it is passive. In turn, according to Lemma 1, $\mathcal{Z}^{\prime}$ is passive if and only if it is positive real, which can be verified through Definition 2.

From the above, we conclude that the original nonreciprocal $n$-port network with impedance matrix $\mathcal{Z}$ is absolutely stable if and only if the equivalent reciprocal $n$-port network's impedance matrix $\mathcal{Z}^{\prime}$ is positive real. Obviously, the Hermitian part of $Z^{\prime}$ is a real symmetric matrix. In this context, it is straightforward to show that Conditions C and D in Theorem 1 are the same as Conditions i) and ii) in Definition 2. Also, according to Condition iii) of Definition 2, the Hermitian part

$$
\mathcal{Z}^{\prime}(j \omega)+\mathcal{Z}^{\prime T}(-j \omega)=2 \operatorname{Re}\left(\mathcal{Z}^{\prime}(j \omega)\right)
$$

needs to be positive semidefinite for the $n$-port network with impedance matrix $\mathcal{Z}$ to be absolutely stable. Using Property 2 [28], and simplifying the conditions by

$$
\left(\operatorname{Re}\left(\sqrt{z_{i, j} z_{j, i}}\right)\right)=\sqrt{\frac{\left|z_{i, j} z_{j, i}\right|+\operatorname{Re}\left(z_{i, j} z_{j, i}\right)}{2}}
$$

where $i, j=1,2, \cdots, m \times n$, we arrive at conditions (15a)(15c). This concludes the proof.

Remark 1. Theorem 1 holds not only for the impedance matrix of a general network but also for its other immittance (admittance, hybrid, and transmission) matrices. The reason for this is that according to Property 1, a gyration operators transform one immittance matrix to another and preserves passivity.

Remark 2. When $m=1$ and $n=2$, Theorem 1 is the same as Llewellyn's absolute stability criterion. Also, for the case $m=1$ and $n \geq 2$, the matrix $\mathbf{Z}_{i j}$ will reduce to a scalar, which is always symmetric, meaning that Condition B of Theorem 1 is always satisfied.

\section{Case Study 1: Absolute Stability of a 1-DOF TRILATERAL HAPTIC SYSTEM}

In this section, the aim is to apply the proposed absolute stability criterion for a 1-DOF trilateral haptic system. In the following, we begin by reviewing a dual-user teleoperation system in position-position control structure.

\section{A. A 1-DOF dual-user teleoperation system}

In a linear time-invariant (LTI) 1-DOF dual-user teleoperation control system, the goal is that two users collaboratively control a robot to perform a desired task on a remote environment. Such a system consists of two master robots for the two users and one slave robot that is in contact with the environment. As elaborated by [15], a Masters Correspondence with Environment Transfer (MCET) dual-user teleoperation system creates for training purposes a correspondence between the masters positions while transferring the environment dynamics to the two users. As seen in Figure 3, this is done via a weight parameter $\alpha \in[0,1]$ that specifies the relative authority of each operator over the slave robot's reference position.

In such a system, the dynamics of the two masters and the slave in contact with the two users and the environment are

$$
\begin{aligned}
z_{m i} v_{h i} & =f_{h i}+f_{c m i} \\
z_{s} v_{e} & =f_{e}+f_{c s}
\end{aligned}
$$

where $z_{m i}(i=1,2)$ and $z_{s}$ are the impedances of the two masters and the slave, respectively. Also, $f_{h i}$ denotes the interaction force between each user and its corresponding master and $f_{e}$ denotes the interaction force between the slave and the environment. Lastly, $v_{h i}$, and $v_{e}$ are the users and the environment velocities and $f_{c m i}$ and $f_{c s}$ are the control signals for the two masters and the slave, respectively.

Since in (21) the impedances relate force to velocity (and not position), modeling each robot by a mass-spring-damper results in $z_{m i}=\frac{m_{m i} s^{2}+b_{m i} s+k_{m i}}{s}$ and $z_{s}=\frac{m_{s} s^{2}+b_{s} s+k_{s}}{s}$. For 


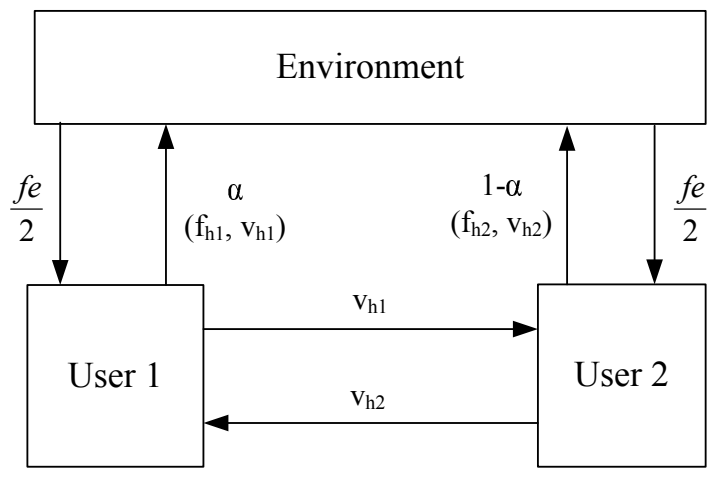

Figure 3. Masters Correspondence with Environment Transfer (MCET) architecture dual-user haptic teleoperation.

this dual-user teleoperation system, the four-channel control laws can be written as [15]:

$$
\begin{aligned}
f_{c m i} & =-c_{m i} v_{h i}-c_{4 m i} v_{h i d}+c_{6 m i} f_{h i}-c_{2 m i} f_{h i d} \\
f_{c s} & =-c_{s} v_{e}+c_{1} v_{e d}-c_{5} f_{e}+c_{3} f_{e d}
\end{aligned}
$$

where $c_{m i}$ and $c_{s}$ are local position controllers, $c_{6 m i}$ and $c_{5}$ are local force controllers, and $c_{1}, c_{2 m i}, c_{3}$, and $c_{4 m i}$ are feedforward and feedback compensators. Also, $v_{\text {hid }}$ and $v_{e d}$ are reference velocities and $f_{\text {hid }}$ and $f_{\text {ed }}$ are references forces for the two masters and the slave selected according to

$$
\begin{aligned}
& v_{h 1 d}=v_{h 2}, \quad v_{h 2 d}=v_{h 1} \\
& v_{e d}=\alpha v_{h 1}+(1-\alpha) v_{h 2}, \quad f_{h 1 d}=\frac{1}{2} f_{e} \\
& f_{h 2 d}=\frac{1}{2} f_{e}, \quad f_{e d}=\alpha f_{h 1}+(1-\alpha) f_{h 2}
\end{aligned}
$$

For simplicity, let us consider the position-position control laws as a special case of the above four-channel control. We will have $c_{1}=c_{s}, c_{4 m 1}=-c_{m 1}$, and $c_{4 m 2}=-c_{m 2}$. Also, $c_{3}, c_{5}, c_{2 m i}$, and $c_{6 m i}$ are zero. Also, let us model each robot by a mass only. Again, since in (21) the impedances relate force to velocity, normally PD position controllers show up as PI velocity controllers:

$$
\begin{aligned}
c_{m 1} & =\frac{k_{p m 1}+k_{v m 1} s}{s}, \quad c_{m 2}=\frac{k_{p m 2}+k_{v m 2} s}{s} \\
c_{s} & =\frac{k_{p s}+k_{v s} s}{s}
\end{aligned}
$$

To get the impedance matrix of position-position control dualuser teleoperation system, first substitute (23) in (22) and then substitute the result in (21) to get

$$
\left[\begin{array}{c}
f_{h 1} \\
f_{h 2} \\
f_{e}
\end{array}\right]=\left[\begin{array}{ccc}
c_{m 1}+z_{m 1} & c_{4 m 1} & 0 \\
c_{4 m 2} & c_{m 2}+z_{m 2} & 0 \\
-\alpha c_{1} & -(1-\alpha) c_{1} & c_{s}+z_{s}
\end{array}\right]\left[\begin{array}{c}
v_{h 1} \\
v_{h 2} \\
v_{e}
\end{array}\right]
$$

Now, let us perform the stability analysis via Theorem 1, where $m=1$ and $n=3$. Evidently, for the impedance matrix (25), the symmetrization condition $\mathrm{A}$ and $\mathrm{B}$ of Theorem 1 holds for any $\alpha$ because $z_{13} z_{21} z_{32}-z_{12} z_{23} z_{31}$ is identical to zero. All poles from elements of (25) are equal to zero.
Table I

THE CONTROLLERS GAINS OF THE POSITION-POSITION DUAL-USER TELEOPERATION SYSTEM USED IN SIMULATIONS.

\begin{tabular}{l|ll|ll|ll}
\hline & \multicolumn{2}{|c|}{ Master \#1 } & \multicolumn{2}{c|}{ Master \#2 } & \multicolumn{2}{c}{ Slave } \\
\hline (A) & $k_{p m 1}$ & 160 & $k_{p m 2}$ & 120 & $k_{p s}$ & 150 \\
& $k_{v m 1}$ & 64 or 80 & $k_{v m 2}$ & 48 & $k_{v s}$ & 85 \\
\hline
\end{tabular}

Analysis of the residues leads to

$$
\begin{aligned}
& k_{11}=k_{p m 1} \geq 0 \\
& k_{22}=k_{p m 2} \geq 0 \\
& k_{33}=k_{p s} \geq 0 \\
& k_{11} k_{22}-k_{12} k_{21}=0 \\
& k_{11} k_{33}-k_{13} k_{31}=0 \\
& k_{22} k_{33}-k_{23} k_{32}=k_{p m 1} k_{p s} \geq 0 \\
& k_{11} k_{22} k_{33}-k_{11} k_{23} k_{32}-k_{22} k_{13} k_{33}-k_{33} k_{12} k_{21} \\
& +k_{13} k_{21} k_{32}+k_{12} k_{23} k_{31}=0
\end{aligned}
$$

Conditions (26)-(32) are satisfied if we choose the proportional control gains to be nonnegative, thus, Conditions $\mathrm{C}$ and $\mathrm{D}$ of Theorem 1 are readily fulfilled. With $s=j \omega$, it is possible to see that the conditions (15a)-(15c) become

$$
\begin{aligned}
& k_{v m 1} \geq 0 \\
& k_{v m 2} \geq 0 \\
& k_{v s} \geq 0 \\
& -\left(k_{v m 1} k_{p m 2}-k_{p m 1} k_{v m 2}\right)^{2} \geq 0 \\
& k_{v m 1} k_{v s} \geq 0 \\
& k_{v m 2} k_{v s} \geq 0 \\
& -k_{v s}\left(k_{v m 1} k_{p m 2}-k_{p m 1} k_{v m 2}\right)^{2} \geq 0
\end{aligned}
$$

Clearly, condition (36) and (39) will be fulfilled for all frequencies $\omega$ if we choose the derivative control gains to be nonnegative and

$$
\frac{k_{p m 1}}{k_{v m 1}}=\frac{k_{p m 2}}{k_{v m 2}}
$$

So, a necessary and sufficient, frequency-independent, and compact condition for absolute stability of the above-described position-position dual-user teleoperation systems is given by (40), where all control gains are nonnegative. Note that the ratios in (40) are merely artifacts of our presentation of the absolute stability conditions meaning that division by zero can be avoided.

\section{B. Simulations}

The position-position dual-user teleoperation system has been simulated in MATLAB/Simulink. There is no time delay in the communication channel between the masters and the slave. Three 1-DOF robots as the two masters and the slave are modeled by masses $m_{m 1}=0.7, m_{m 2}=0.5$, and $m_{s}=1.5$, respectively. According to (40), the stability of the positionposition dual-user teleoperation system should depend on the controllers gains. In the simulations, the controllers gains $k_{p m 1}, k_{v m 1}, k_{p m 2}, k_{v m 2}, k_{p s}$, and $k_{v s}$ were chosen according to Table I. Evidently, (40) is satisfied for $k_{v m 1}=64$ and violated for $k_{v m 1}=80$. Also, $\alpha \in[0,1]$.

In simulations, to check the absolute stability of the threeport network, the master \#2 and the slave ports are connected to LTI terminations with transfer functions $\frac{1}{s+1}$, which are 


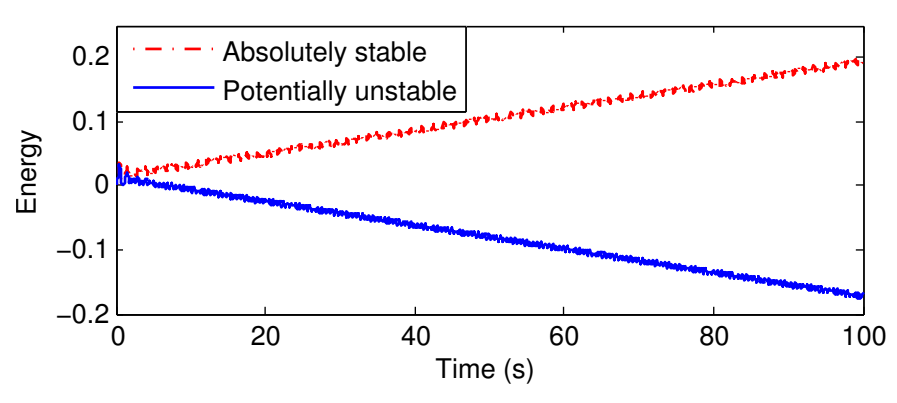

Figure 4. Simulation results for the dual-user teleoperation system. Input energy at the master \#1's port is shown. Simulation parameters are listed in Table I for the absolute stability case with $k_{v m 1}=64$, and for the potentially unstable case with $k_{v m 1}=80$

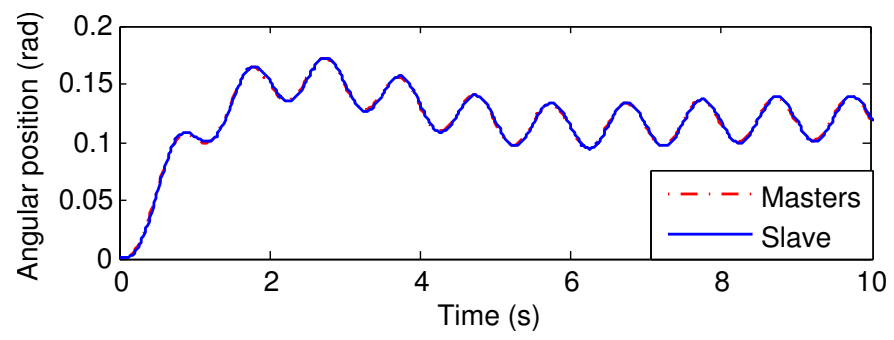

Figure 5. Simulation results for the dual-user teleoperation system. The desired and actual positions for the slave are shown. A sinusoidal force was applied to the master \#1 while the master \#2 and the slave were connected to passive terminations. Simulation parameters are listed in Table I for the absolutely stable case of $k_{v m 1}=64$.

passive because, for $s=j \omega$, we have $\operatorname{Re}\left(\frac{1}{\mathrm{~s}+1}\right)=\frac{1}{\omega^{2}+1}>0$. Port 1 is open and a sine-wave input $f_{h 1}$ is applied to the master \#1. The input energy $E_{s}(t)$ in (6) is plotted in Figure 4 for $k_{v m 1}=64$ and $k_{v m 1}=80$ while the rest of the control gains are listed in Table I. As it can be seen, if the control gains are selected according to (40), e.g., as listed in Table I with $k_{v m 1}=64$, then the input energy at port 1 is non-negative at all times, indicating the absolute stability of the trilateral haptic system. However, when we change $k_{v m 1}$ to 80 , which violates (40), the input energy $E_{s}(t)$ will become negative at least for a period of time, indicating potential instability of the trilateral haptic system. For the case of $k_{v m 1}=64$, Figure 5 depicts the linear combination of the two masters positions based on authority factor $\alpha$ (i.e., the desired position for the slave) versus the slave position. The above show that there is agreement between the theoretical absolute stability condition (40) and the simulations.

\section{Case Study 2: Absolute Stability of a 2-DOF BILATERAL HAPTIC SYSTEM}

In this section, the aim is to apply the proposed absolute stability criterion to a coupled 2-DOF bilateral haptic teleoperation system. Then, experiments will be conducted for verifying the theoretical absolute stability conditions.

\section{A. A 2-DOF bilateral teleoperation system}

In a 2-DOF LTI bilateral teleoperation system, the dynamics of the master and the slave in contact with the user and the environment, respectively, are

$$
\begin{aligned}
\mathbf{Z}_{m} V_{h} & =F_{h}+F_{c m} \\
\mathbf{Z}_{s} V_{e} & =F_{e}+F_{c s}
\end{aligned}
$$

where $\mathbf{Z}_{m}=\frac{\mathbf{M}_{m} s^{2}+\mathbf{B}_{m} s+\mathbf{K}_{m}}{s}$, and $\mathbf{Z}_{s}=\frac{\mathbf{M}_{s} s^{2}+\mathbf{B}_{s} s+\mathbf{K}_{s}}{s}$, are $2 \times 2$ impedance $\stackrel{s}{\text { matrices }}$ of the master and the slave, respectively. Assume

$$
\begin{aligned}
\mathbf{M}_{i} & =\left[\begin{array}{ll}
m_{i y y} & m_{i y z} \\
m_{i y z} & m_{i z z}
\end{array}\right], \quad \mathbf{B}_{i}=\left[\begin{array}{ll}
b_{i y y} & b_{i y z} \\
b_{i y z} & b_{i z z}
\end{array}\right], \\
\mathbf{K}_{i} & =\left[\begin{array}{ll}
k_{i y y} & k_{i y z} \\
k_{i y z} & k_{i z z}
\end{array}\right]
\end{aligned}
$$

where $i=m, s$ correspond to the master and the slave, respectively. The subscripts are chosen to correspond to the $y-z$ plane while any other plane can be chosen. Also, $F_{h}=\left[f_{h y}, f_{h z}\right]^{T}$ denotes the interaction force vector between the user and the master and $F_{e}=\left[f_{e y}, f_{e z}\right]^{T}$ denotes the interaction force vector between the slave and the environment. Lastly, $V_{h}=\left[v_{h y}, v_{h z}\right]^{T}$ and $V_{e}=\left[v_{e y}, v_{e z}\right]^{T}$ are the user and the environment velocities.

Similar to the previous case study, let us consider positionposition control laws [29]:

$$
\begin{aligned}
F_{c m} & =-\mathbf{C}_{m} V_{h}+\mathbf{C}_{m} V_{e} \\
F_{c s} & =-\mathbf{C}_{s} V_{e}+\mathbf{C}_{s} V_{h}
\end{aligned}
$$

where the normally PD position controllers show up as PI velocity controllers:

$$
\begin{aligned}
\mathbf{C}_{m} & =\left[\begin{array}{ll}
\frac{k_{p m y y}+k_{v m y y} s}{s} & \frac{k_{p m y z}+k_{v m y z} s}{s} \\
\frac{k_{p m z y}+k_{v m z y} s}{s} & \frac{k_{p m z z}+k_{v m z z} s}{s}
\end{array}\right] \\
\mathbf{C}_{s} & =\left[\begin{array}{ll}
\frac{k_{p s y y}+k_{v s y y} s}{s} & \frac{k_{p s y z}+k_{v s y z} s}{s} \\
\frac{k_{p s z y}+k_{v s z y} s}{s} & \frac{k_{p s z z}+k_{v s z z} s}{s}
\end{array}\right]
\end{aligned}
$$

By substituting (42) in (41), the impedance matrix representation of the 2-DOF teleoperator is found as

$$
\left[\begin{array}{c}
F_{h} \\
F_{e}
\end{array}\right]=\left[\begin{array}{cc}
\mathbf{C}_{m}+\mathbf{Z}_{m} & -\mathbf{C}_{m} \\
-\mathbf{C}_{s} & \mathbf{C}_{s}+\mathbf{Z}_{s}
\end{array}\right]\left[\begin{array}{c}
V_{h} \\
V_{e}
\end{array}\right]
$$

Now, let us investigate the absolute stability of the teleoperator via Theorem 1 for the case of $m=2$ and $n=2$. With $s=j \omega$, the symmetrization conditions of A and B boils down to the following four conditions involving the control gains and the frequency $\omega$ :

$$
\begin{aligned}
& k_{v m y z}-k_{v m z y}+\frac{j\left(k_{p m z y}-k_{p m y z}\right)}{\omega}=0 \\
& k_{v s y z}-k_{v s z y}+\frac{j\left(k_{p s z y}-k_{p s y z}\right)}{\omega}=0 \\
& \omega^{2}\left(k_{v m y z} k_{v s y y}-k_{v s y z} k_{v m y y}\right)+j \omega\left(k_{v s y z} k_{p m y y}\right. \\
& \left.+k_{p s y z} k_{v m y y}-k_{v m y z} k_{p s y y}-k_{p m y z} k_{v s y y}\right) \\
& +k_{p s y z} k_{p m y y}-k_{p m y z} k_{p s y y}=0 \\
& \omega^{2}\left(k_{v m z z} k_{v s y z}-k_{v s z z} k_{v m y z}\right)+j \omega\left(k_{v s z z} k_{p m y z}\right. \\
& \left.+k_{p s z z} k_{v m y z}-k_{v m z z} k_{p s y z}-k_{p m z z} k_{v s y z}\right) \\
& +k_{p s z z} k_{p m y z}-k_{p m z z} k_{p s y z}=0
\end{aligned}
$$

Conditions (45) will be fulfilled for all frequencies $\omega$ if the gains of the PD controllers (43) satisfy

$$
\begin{aligned}
& k_{p m y z}=k_{p m z y}, \quad k_{v m y z}=k_{v m z y} \\
& k_{p s y z}=k_{p s z y}, \quad k_{v s y z}=k_{v s z y} \\
& \frac{k_{v m y y}}{k_{v s y y}}=\frac{k_{v m y z}}{k_{v s y z}}=\frac{k_{p m y y}}{k_{p s y y}}=\frac{k_{p m y z}}{k_{p s y z}}=\frac{k_{v m z z}}{k_{v s z z}}=\frac{k_{p m z z}}{k_{p s z z}}
\end{aligned}
$$


It is easy to see that, under (46), all the elements of the impedance matrix (44) have only a simple pole on the imaginary axis, thus satisfying Condition C. Define $k_{i j}, i, j=1,2,3$ as the elements of residues matrix $K$, analysis of the residues according to Condition D leads to the following constraints:

$$
\begin{aligned}
& k_{m y y}+k_{\text {pmyy }} \geq 0 \\
& k_{m z z}+k_{p m z z} \geq 0 \\
& k_{s y y}+k_{p s y y} \geq 0 \\
& k_{s z z}+k_{p s z z} \geq 0 \\
& Q_{m}+Q_{p m}+W_{m p m} \geq 0 \\
& Q_{m}\left(k_{s y y}+k_{p s y y}\right)+Q_{p m}\left(k_{s y y}+k_{m y y}\right) \\
& +k_{s y y} W_{m p m} \geq 0 \\
& Q_{m}\left(k_{s z z}+k_{p s z z}\right)+Q_{p m}\left(k_{s z z}+k_{m z z}\right) \\
& +k_{s z z} W_{m p m} \geq 0 \\
& Q_{s}\left(Q_{m}+Q_{p m}+W_{m p m}\right)+Q_{m}\left(Q_{p s}+W_{s p s}\right) \\
& +\frac{k_{p m y y}}{k_{p s y y}} Q_{p s} W_{m s} \geq 0
\end{aligned}
$$

where $W_{m p m}=k_{m y y} k_{p m z z}+k_{m z z} k_{p m y y}-2 k_{m y z} k_{p m y z}$, $W_{s p s}=k_{s y y} k_{p s z z}+k_{s z z} k_{p s y y}-2 k_{s y z} k_{p s y z}, W_{m s}=$ $k_{m y y} k_{s z z}+k_{m z z} k_{s y y}-2 k_{m y z} k_{s y z}$. Also, $Q_{i}=k_{i y y} k_{i z z}-$ $k_{i y z}^{2}$, where $i=m, s, p m, p s$. It is easy to see that, the condition set (47) holds if

$$
k_{i y y} k_{i z z} \geq k_{i y z}^{2}, \quad i=m, s, p m, p s
$$

Now, let us deal with Condition E of Theorem 1. Condition (15a) turns out to state

$$
\begin{aligned}
& b_{m y y}+k_{v m y y} \geq 0 \\
& b_{m z z}+k_{v m z z} \geq 0 \\
& b_{s y y}+k_{v s y y} \geq 0 \\
& b_{s z z}+k_{v s z z} \geq 0
\end{aligned}
$$

Under (46) and (48), the second principal minor condition, i.e., (15b), gives

$$
\begin{aligned}
& U_{m}+Q_{v m}+b_{m y y} k_{v m z z}+b_{m z z} k_{v m y y} \geq 0 \\
& U_{s}+Q_{v s}+b_{s y y} k_{v s z z}+b_{s z z} k_{v s y y} \geq 0
\end{aligned}
$$

where $U_{m}=b_{m y y} b_{m z z}-b_{m y z}^{2}, U_{s}=b_{s y y} b_{s z z}-b_{s y z}^{2}$, $Q_{v s}=k_{v s y y} k_{v s z z}-k_{v s y z}^{2}$, and $Q_{v m}=k_{v m y y} k_{v m z z}-k_{v m y z}^{2}$. Similarly, the third principal minor condition requires

$$
\begin{aligned}
& U_{m}\left(k_{v s y y}+b_{s y y}\right)+\frac{b_{m y y} k_{v m y y} Q_{v m}}{k_{v s y y}}+b_{s y y} W_{m v m} \geq 0 \\
& U_{s}\left(k_{v m y y}+b_{m y y}\right)+\frac{b_{s y y} k_{v s y y} Q_{v s}}{k_{v m y y}}+b_{m y y} W_{s v s} \geq 0
\end{aligned}
$$

where $W_{m v m}=k_{m y y} k_{v m z z}+k_{m z z} k_{v m y y}-2 k_{m y z} k_{v m y z}$ and $W_{s v s}=k_{s y y} k_{v s z z}+k_{s z z} k_{v s y y}-2 k_{s y z} k_{v s y z}$. Finally, the fourth principal minor condition, i.e., (15c), mandates

$$
U_{s}\left(U_{m}+Q_{s} \frac{k_{v m y y}^{2}}{k_{v s y y}^{2}}\right)+Q_{v s}\left(U_{m}+\frac{k_{v m y y}}{k_{v s y y}}\right) \geq 0
$$

All in all, one can see that conditions (49)-(54) will be fulfilled for all frequencies $\omega$ if

$$
\begin{aligned}
& b_{m y y} b_{m z z} \geq b_{m y z}^{2}, \quad k_{v m y y} k_{v m z z} \geq k_{v m y z}^{2} \\
& b_{s y y} b_{s z z} \geq b_{s y z}^{2}, \quad k_{v s y y} k_{v s z z} \geq k_{v s y z}^{2}
\end{aligned}
$$

Table II

THE CONTROLLERS GAINS OF THE 2-DOF BILATERAL TELEOPERATION SYSTEM USED IN EXPERIMENTS. (A1) AND (A2) ABSOLUTELY STABLE, (B) Potentially unstable.

\begin{tabular}{l|llll}
\hline & & $\mathrm{A} 1$ & $\mathrm{~A} 2$ & $\mathrm{~B}$ \\
\hline \hline \multirow{5}{*}{ Master } & $k_{\text {pmyy }}$ & 500 & 1000 & 500 \\
& $k_{\text {vmyy }}$ & 300 & 800 & 300 \\
& $k_{\text {pmzz }}$ & 500 & 800 & 500 \\
& $k_{\text {vmzz }}$ & 300 & 600 & 250 \\
& $k_{\text {pmyz }}$ & 450 & 50 & 550 \\
& $k_{\text {vmyz }}$ & 250 & 25 & 300 \\
\hline \multirow{5}{*}{ Slave } & $k_{\text {psyy }}$ & 500 & 1000 & 500 \\
& $k_{\text {vsyy }}$ & 300 & 800 & 300 \\
& $k_{\text {pszz }}$ & 500 & 800 & 500 \\
& $k_{\text {vszz }}$ & 300 & 600 & 250 \\
& $k_{\text {psyz }}$ & 450 & 50 & 550 \\
& $k_{\text {vsyz }}$ & 250 & 25 & 300 \\
\hline
\end{tabular}

So, a sufficient, frequency-independent, and compact condition set for absolute stability of the above-described 2-DOF bilateral teleoperator is

$$
\begin{aligned}
& b_{m y y} b_{m z z} \geq b_{m y z}^{2}, \quad b_{s y y} b_{s z z} \geq b_{s y z}^{2} \\
& k_{i y y} k_{i z z} \geq k_{i y z}^{2}, \quad i=m, s, p m, p s, v m, v s \\
& k_{p m y z}=k_{p m z y}, \quad k_{v m y z}=k_{v m z y} \\
& k_{p s y z}=k_{p s z y}, \quad k_{v s y z}=k_{v s z y} \\
& \frac{k_{v m y y}}{k_{v s y y}}=\frac{k_{v m y z}}{k_{v s y z}}=\frac{k_{p m y y}}{k_{p s y y}}=\frac{k_{p m y z}}{k_{p s y z}}=\frac{k_{v m z z}}{k_{v s z z}}=\frac{k_{p m z z}}{k_{p s z z}}
\end{aligned}
$$

where all control gains are nonnegative. Again, the ratios in (56) are merely artifacts of our presentation of the absolute stability conditions meaning that division by zero can be avoided. An alternative to the above stability analysis is to use the extended Z-W criterion [30]. However, in that approach, the stability conditions often need to be evaluated numerically rather than being in closed form.

\section{B. Experiments}

We use a 2-DOF bilateral teleoperation system comprising two 3-joint Phantom Premium 1.5A robots (Sensable Technologies/Geomagic, Wilmington, MA) as the master and as the slave. Out of the three joints of each robot, the second $(y)$ and the third $(z)$ joints, which form a vertical plane, are considered. The first joint $(x)$, which corresponds to rotation of the vertical plane about an axis, is locked using highgain control. The experimental setup is shown in Figure 6, where a human user interacts with the master while the slave is physically connected via a $2 \mathrm{D}$ passive spring array to a stiff wall. The stiffness of the springs were chosen such that sufficient displacements result as the robot end-effector applies forces. Given the limited maximum continuous output force of $1.4 \mathrm{~N}$ of the Phantom Premium the stiffness was selected to be $45 \mathrm{~N} / \mathrm{m}$. Even though we will only implement positionposition teleoperation control, the master is equipped with a JR3 6-DOF force/torque sensor (product model: 50M31, JR3, Inc., Woodland, CA) for measuring the applied forces such that $E_{s}(t)$ in (6) can be quantified. With knowledge of Phantom 1.5A robot dynamics from [31], gravity compensation for each robot arm is performed by calculating the gravity vector at each point within the workspace and feeding it forward. All data logging and robot control actions occurred with a $1 \mathrm{kHz}$ sampling frequency.

According to the condition set (56), the absolute stability of the 2-DOF bilateral teleoperator should depend on the control 


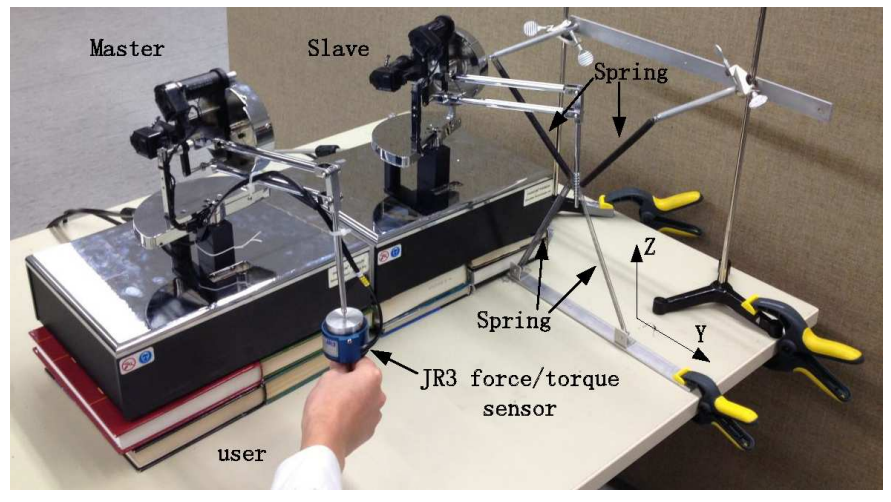

Figure 6. Experimental setup where the master is controlled by a human user and the slave is physically connected via a $2 \mathrm{D}$ passive spring array to a stiff wall.

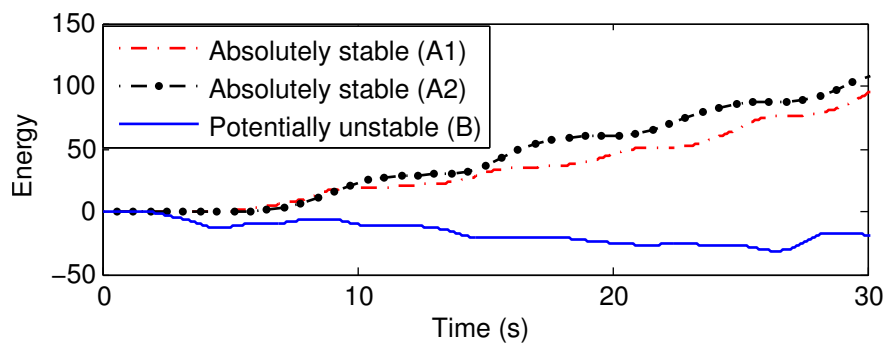

Figure 7. Experimental results for the 2-DOF bilateral teleoperation system. Input energy at the master's port is shown while the slave is physically connected via a $2 \mathrm{D}$ passive spring array to a stiff wall. The control gains are listed in Table $\mathrm{II}(\mathrm{A} 1)$ and (A2) for the absolutely stable case and in Table II(B) for the potentially unstable case.

gains. In the experiments, the control gains were chosen according to either case A1, A2 or case B listed in Table II. For these cases, the input energy $E_{s}(t)$ in (6) is plotted in Figure 7. As it can be seen, if the control gains are selected according to (56), i.e., as listed in Table II(A1) and (A2), then the input energy $E_{s}(t)$ in (6) at the master's port are non-negative at all times, indicating the absolute stability of the bilateral teleoperator. However, when the control gains are selected as Table II(B), which violates (56), the input energy $E_{s}(t)$ in (6) will become negative at least for a period of time, indicating the potential instability of the bilateral teleoperator.

Figure 8 and 9 depicts the master position versus the slave position for each of the two joints for the parameters listed in Table II(A1) and (A2). The above show that there is agreement between the theoretical absolute stability condition (56) and the experiments. Note that as long as the control gains satisfy (56), the system will be stable. However, the exact values of such stabilizing control gains influence position tracking performance shown in Figure 8 and 9. Also, the force tracking performance is shown in Figure 10, where the master-side forces are measured by a JR3 force sensor, while the slaveside forces are calculated through multiplying the stiffness of the springs by the slave robot's displacement.

\section{CONCLUSIONS AND Future WORKS}

In the beginning of this paper, it was shown via an example that applying Llewellyn's absolute stability criterion once in each DOF of a multi-DOF bilateral haptic system cannot guarantee the absolute stability as this method ignores the coupling between DOFs that may exist in the system. Also, reducing a multi-lateral haptic system to a bilateral
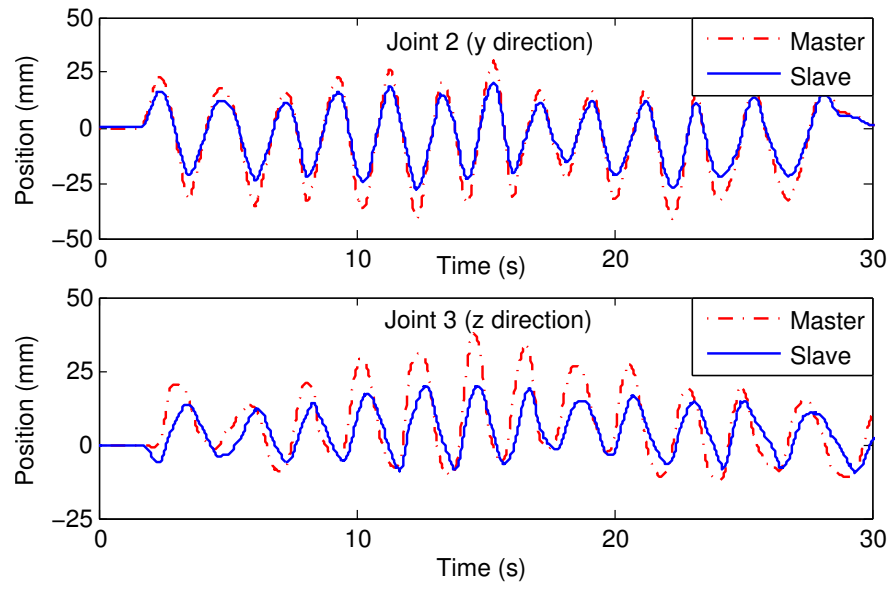

Figure 8. Experimental results for the 2-DOF bilateral teleoperation system. The master and slave positions in terms of their second and the third joints are shown when using the control gains listed in Table II(A1), which amount to absolute stability of the bilateral teleoperator.
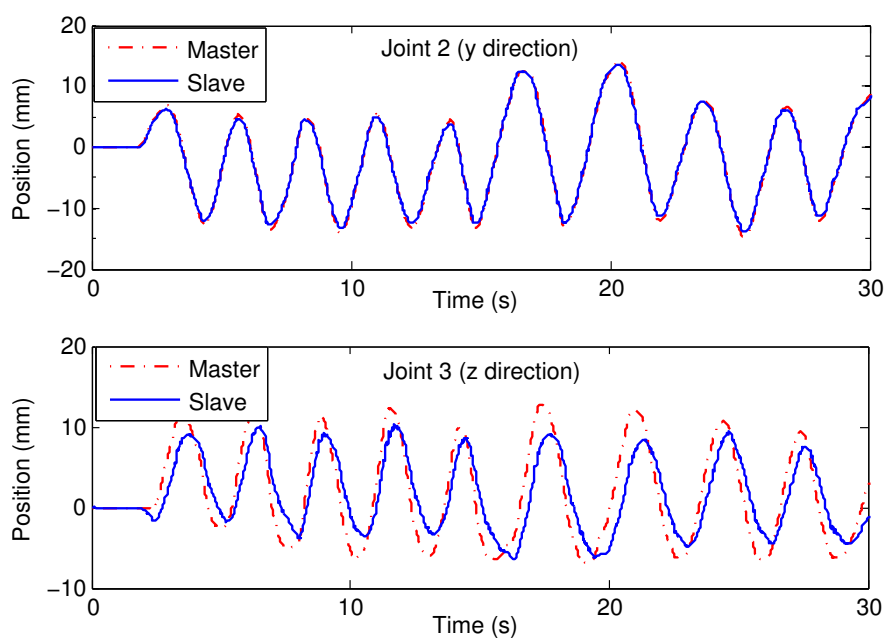

Figure 9. Experimental results for the 2-DOF bilateral teleoperation system. The master and slave positions in terms of their second and the third joints are shown when using the control gains listed in Table II(A2), which amount to absolute stability of the bilateral teleoperator.

haptic system by terminating some of its terminals such that Llewellyn's absolute stability criterion can be used is cumbersome. This paper presented a closed-form and easyto-use absolute stability criterion for multi-DOF multi-lateral haptic systems. Through two case studies, we elaborated on its application in absolute stability analysis of a 1-DOF trilateral and a 2-DOF bilateral haptic system. Through simulations and experiments, the proposed absolute stability criterion was validated. In the future, a possible investigation is the stability-transparency trade-offs for haptic systems in light of the proposed stability criterion. Also, investigating the stability of multi-dof multi-lateral haptic systems in which multiple master robots control a higher-DOF slave robot for performing a dexterous task through collaboration of several human operators is an interesting direction for future research.

\section{APPENDIX}

Llewellyn's criterion: If $p_{m n}=r_{m n}+j x_{m n}, m, n=1,2$, represents any of the four immittance parameters $(z, y, h$, and $g$ ) of a two-port network, for all real values of frequencies $\omega$, the network is absolutely stable if and only if 

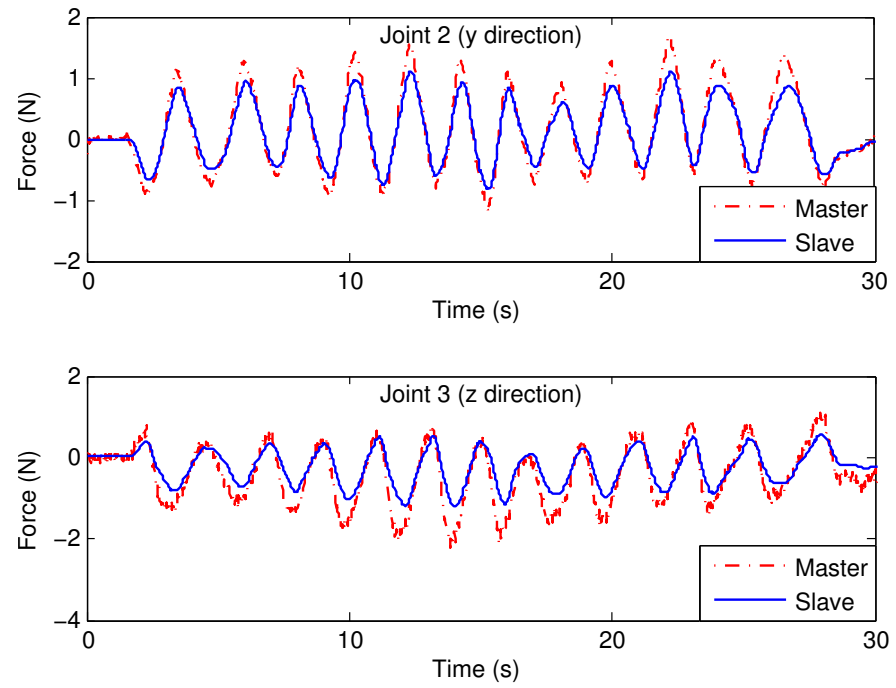

Figure 10. Experimental results for the 2-DOF bilateral teleoperation system. The master and slave forces in terms of their second and the third joints are shown when using the control gains listed in Table II(A2), which amount to absolute stability of the bilateral teleoperator.

1) The $P$ matrix elements have no poles in the right-half plane (RHP).

2) Any poles of the $P$ matrix elements on the imaginary axis are simple with residues that satisfy

$$
\begin{aligned}
& k_{m m} \geq 0, \quad m=1,2 \\
& k_{11} k_{22}-k_{12} k_{21} \geq 0, \quad k_{12}=k_{21}^{*}
\end{aligned}
$$

where $k_{m n}$ denotes the residue of $p_{m n}$ and $k_{m n}^{*}$ is the complex conjugate of $k_{m n}$.

3) The real and imaginary parts of the $P$ matrix elements satisfy

$$
\begin{aligned}
& r_{11} \geq 0 \\
& r_{22} \geq 0 \\
& r_{11} r_{22}-\frac{\left|p_{12} p_{21}\right|+\operatorname{Re}\left(p_{12} p_{21}\right)}{2} \geq 0
\end{aligned}
$$

\section{REFERENCES}

[1] S. Nudehi, R. Mukherjee, and M. Ghodoussi, "A shared-control approach to haptic interface design for minimally invasive telesurgical training," IEEE Transactions on Control Systems Technology, vol. 13, no. 4, pp. 588-592, July 2005.

[2] Y. Ye and P. Liu, "Improving haptic feedback fidelity in wave-variablebased teleoperation orientated to telemedical applications," IEEE Transactions on Instrumentation and Measurement, vol. 58, no. 8, pp. 2847 -2855, August 2009.

[3] D. J. Lee and M. W. Spong, "Semi-Autonomous Teleoperation of Multiple Cooperative Robots for Human-Robot Lunar Exploration," 2006 AAAI Spring Symposia, no. 1, pp. 1-8, 2006.

[4] W. T. Lo, Y. Liu, I. Elhajj, N. Xi, Y. Wang, and T. Fukuda, "Cooperative teleoperation of a multirobot system with force reflection via internet," IEEE/ASME Transactions on Mechatronics, vol. 9, no. 4, pp. $661-670$, December 2004.

[5] I. G. Polushin, S. N. Dashkovskiy, A. Takhmar, and R. V. Patel, "A small gain framework for networked cooperative force-reflecting teleoperation," Automatica, vol. 49, no. 2, pp. 338-348, 2013.
[6] G. Lombardi and B. Neri, "Criteria for the evaluation of unconditional stability of microwave linear two-ports: a critical review and new proof," IEEE Transactions on Microwave Theory and Techniques, vol. 47, no. 6, pp. $746-751$, Jun 1999.

[7] E. Tan, "Simplified graphical analysis of linear three-port stability," IEE Proceedings on Microwaves, Antennas and Propagation, vol. 152, no. 4, pp. 209-213, August 2005.

[8] G. Raisbeck, "A definition of passive linear networks in terms of time and energy," Journal of Applied Physics, vol. 25, no. 12, pp. 1510-1514, December 1954

[9] B. Willaert, B. Corteville, D. Reynaerts, H. V. Brussel, and E. B. V. Poorten, "A mechatronic analysis of the classical position-force controller based on bounded environment passivity," The International Journal of Robotics Research, pp. 1 - 18, August 2010.

[10] M. Shahbazi, H. A. Talebi, and M. J. Yazdanpanah, "A control architecture for dual user teleoperation with unknown time delays: A sliding mode approach," in 2010 IEEE/ASME International Conference on AIM, July 2010, pp. $1221-1226$.

[11] M. Panzirsch, J. Artigas, A. Tobergte, P. Kotyczka, P. Carsten, A. AlbuSchaeffer, and G. Hirzinger, "A peer-to-peer trilateral passivity control for delayed collaborative teleoperation," in Institute of Robotics and Mechatronics, vol. 7282, December 2012, pp. 395-406.

[12] V. Mendez and M. Tavakoli, "A passivity criterion for n-port multilateral haptic systems," in 2010 49th IEEE Conference on Decision and Control $(C D C)$, December 2010, pp. $274-279$.

[13] S. Haykin, Active Network Theory. Addison-Wesley, 1970.

[14] F. Llewellyn, "Some fundamental properties of transmission systems," Proceedings of the IRE, vol. 2, no. 1, pp. 271-283, 1952.

[15] B. Khademian and K. Hashtrudi-Zaad, "Shared control architectures for haptic training: Performance and coupled stability analysis," The International Journal of Robotics Research, vol. 30, pp. 1627-1642, 2011

[16] J. Li, M. Tavakoli, and Q. Huang, "Stability analysis of trilateral haptic collaboration," IEEE World Haptics Conference 2013, pp. 611 -616, April 2013

[17] W. Ku, "Stability of linear active nonreciprocal n-ports," J. Franklin Inst., no. 276, pp. $207-224,1963$.

[18] J. Speich and M. Goldfarb, "An implementation of loop-shaping compensation for multidegree-of-freedom macro-microscaled telemanipulation," IEEE Transactions on Control Systems Technology, vol. 13, no. 3, pp. 459 - 464, May 2005

[19] P. Malysz and S. Sirouspour, "Trilateral teleoperation control of kinematically redundant robotic manipulators," International Journal of Robotics Research, vol. 30, pp. 1643-1664, November 2011.

[20] J.-H. Ryu, C. Preusche, B. Hannaford, and G. Hirzinger, "Time domain passivity control with reference energy following," IEEE Transactions on Control Systems Technology, vol. 13, no. 5, pp. 737 - 742, Sept. 2005.

[21] R. J. Duffin, D. Hazony, and N. Morrison, The Gyration Operator in Network Theory. Scientific Report No. 7, AF 19 (628) 1699, CRST I Sills Bld 5285 Port Royal Road, Springfield, Virginia, 1965.

[22] H. K. Khalil, Nonlinear Systems. Prentice Hall, 2002.

[23] R. A. Horn and C. R. Johanson, Matrix Analysis. Cambridge University Press, 2012.

[24] E. Zeheb and E. Walach, "Necessary and sufficient conditions for absolute stability of linear n-ports," International Journal of Circuit Theory and Applications, vol. 9, no. 3, pp. 311-330, 1981.

[25] D. Youla, "A stability characterization of the reciprocal linear passive n-port," Proc. IRE, vol. 47, pp. 1150-1151, 1959.

[26] D.Youla, "A note on the stability of linear, nonreciprocal n-port," Proc. IRE, vol. 48, pp. 121-122, 1960.

[27] H. J. Marquez, Nonlinear Control Systems Analysis and Design. Wiley, 2003.

[28] K. N. Swamy, "On sylvester's criterion for positive-semidefinite matrices," IEEE Transactions on Automatic Control, vol. 18, no. 3, pp. 306-306, 1973.

[29] M. Tavakoli, A. Aziminejad, R. Patel, and M. Moallem, "High-fidelity bilateral teleoperation systems and the effect of multimodal haptics," IEEE Transactions on Systems, Man, and Cybernetics, Part B: Cybernetics, vol. 37, no. 6, pp. 1512 -1528, December 2007.

[30] K. Razi and K. Hashtrudi-Zaad, "Extension of zeheb-walach absolute stability criteria for robot-human interactions," 51st IEEE Conference on Decision and Control, pp. 1186 - 1191, December 2012.

[31] M. C. avusoglu, M. C. avusoglu, D. Feygin, and F. Tendick, "A critical study of the mechanical and electrical properties of the phantom haptic interface and improvements for high performance control," Presence, vol. 11 , pp. 555-568, 2002 . 